\title{
Panventriculomegaly with a wide foramen of Magendie and large cisterna magna
}

\author{
Hiroshi Kageyama, MD, ${ }^{1-3}$ Masakazu Miyajima, MD, PhD, ${ }^{1}$ Ikuko Ogino, BPHARM, ${ }^{1}$ \\ Madoka Nakajima, MD, PhD, ${ }^{1}$ Kazuaki Shimoji, MD, PhD, ${ }^{1}$ Ryoko Fukai, MD, PhD, ${ }^{2}$ \\ Noriko Miyake, MD, PhD, ${ }^{2}$ Kenichi Nishiyama, MD, PhD, ${ }^{4}$ Naomichi Matsumoto, MD, PhD, ${ }^{2}$ and \\ Hajime Arai, MD, $\mathrm{PhD}^{1}$
}

\begin{abstract}
'Department of Neurosurgery, Graduate School of Medicine, Juntendo University, Tokyo; 'Department of Human Genetics, Yokohama City University Graduate School of Medicine, Yokohama; ${ }^{2}$ Department of Neurosurgery, Kuki General Hospital, Kuki, Saitama; and ${ }^{4}$ Brain Research Institute, University of Niigata, Japan
\end{abstract}

\begin{abstract}
OBJECTIVE The authors' goal in this paper is to provide the first clinical, radiological, and genetic studies of panventriculomegaly (PaVM) defined by a wide foramen of Magendie and large cisterna magna.

METHODS Clinical and brain imaging data from 28 PaVM patients (including 10 patients from 5 families) were retrospectively studied. Five children were included. In adult patients, the age at onset was $56.0 \pm 16.7$ years. Tetraventricular dilation, aqueductal opening with flow void on T2-weighted images, and a wide foramen of Magendie and large cisterna magna (wide cerebrospinal fluid space at the fourth ventricle outlet) were essential MRI findings for PaVM diagnosis. 3D fast asymmetrical spin echo sequences were used for visualization of cistern membranes. Time-spatial labeling inversion pulse examination was performed to analyze cerebrospinal fluid movement. Copy number variations were determined using high-resolution microarray and were validated by quantitative polymerase chain reaction with breakpoint sequencing.
\end{abstract}

RESULTS Adult patients showed gait disturbance, urinary dysfunction, and cognitive dysfunction. Five infant patients exhibited macrocranium. Patients were divided into 2 subcategories, those with or without downward bulging third ventricular floors and membranous structures in the prepontine cistern. Patients with bulging floors were successfully treated with endoscopic third ventriculostomy. Genetic analysis revealed a deletion in DNAH14 that encodes a dynein heavy chain protein associated with motile cilia function, and which co-segregated with patients in a family without a downward bulging third ventricular floor.

CONCLUSIONS Panventriculomegaly with a wide foramen of Magendie and a large cisterna magna may belong to a subtype of congenital hydrocephalus with familial accumulation, younger age at onset, and symptoms of normal pressure hydrocephalus. In addition, a family with PaVM has a gene mutation associated with dysfunction of motile cilia.

http://thejns.org/doi/abs/10.3171/2015.6.JNS15162

KEY WORDS hydrocephalus; congenital; cilia; large cisterna magna

$\mathrm{C}$ ERTAIN congenital anomalies of adult-onset hydrocephalus share clinical symptoms with idiopathic normal pressure hydrocephalus (iNPH), such as long-standing overt ventriculomegaly, aqueductal stenosis,$^{29}$ and Blake's pouch cyst. ${ }^{8}$ However, no disease-associated gene mutations have been identified, including those responsible for these congenital diseases. Moreover, although LICAM mutations can cause aqueductal stenosis, patients carrying these mutations have clinical features different from patients with NPH. ${ }^{1}$

Here, we describe panventriculomegaly (PaVM), a unique clinical entity that is defined by a wide foramen of Magendie and a large cisterna magna. This type of hydrocephalus has not been previously reported. PaVM exhibits

ABBREVIATIONS CNV = copy number variation; DESH = disproportionately enlarged subarachnoid space hydrocephalus; DGV = Database of Genomic Variants; ETV = endoscopic third ventriculostomy; FASE = fast asymmetrical spin echo; $\mathrm{NPH}=$ idiopathic NPH; $\mathrm{LP}=$ lumboperitoneal; $\mathrm{NPH}=$ normal pressure hydrocephalus; $\mathrm{PaVM}=$ panventriculomegaly; $P C R$ = polymerase chain reaction; $q P C R$ = quantitative PCR; time-SLIP = time-spatial labeling inversion pulse; VP = ventriculoperitoneal. SUBMITTED January 23, 2015. ACCEPTED June 3, 2015.

INCLUDE WHEN CITING Published online December 4, 2015; DOI: 10.3171/2015.6.JNS15162. 
several unique characteristics with an apparent congenital etiology. We describe detailed clinical, radiological, and genetic features in 28 patients.

\section{Methods}

\section{Ethics Statement}

The institutional review boards of Juntendo University Hospital and Yokohama City University School of Medicine approved the study protocol. All patients gave their written informed consent to participate in this study.

\section{Diagnostic Criteria and Participants}

Patients were recruited from January 1, 2009, to October 31, 2013. A diagnosis of PaVM was made using MR imaging. The inclusion criteria were 1) tetraventricular dilation, 2) aqueduct opening with flow void on T2-weighted images, and 3) a wide foramen of Magendie and large cisterna magna on MR imaging (wide CSF space at the fourth ventricle outlet).

Twenty-eight patients (16 males and 12 females), including 5 younger than 10 years, with $\mathrm{PaVM}$ incorporating a wide foramen of Magendie and a large cisterna magna, were identified from the Department of Neurosurgery at Juntendo University and other associated hospitals. In adult patients, the age at onset was $56.0 \pm 16.7$ years. Of the total 28 patients, 10 patients were from 5 families (Table 1 and Fig. 1). Two unaffected controls were also included for genetic analysis. Two autopsied brains from another 2 unaffected controls were used for immunostaining.

\section{Clinical Analysis}

Medical records and neuroradiological examinations of all patients were retrospectively analyzed by 2 neurosurgeons (H.K. and M.M.). Clinical characteristics were

TABLE 1. Patients with PaVM with a wide foramen of Magendie and large cisterna magna: characteristics and treatment

\begin{tabular}{|c|c|c|c|c|c|c|c|}
\hline Patient No. & Family: Position & $\begin{array}{l}\text { Sex, Age } \\
\text { (yrs) }\end{array}$ & Symptoms & $\begin{array}{l}\text { Prepontine } \\
\text { Membrane }\end{array}$ & $\begin{array}{l}\text { Surgical } \\
\text { Procedure }\end{array}$ & $\begin{array}{l}\text { CNV Analysis } \\
\text { (gene mutation) }\end{array}$ & Outcome \\
\hline \multicolumn{8}{|c|}{ w/o downward bulging 3rd ventricular floor $(n=11)$} \\
\hline 1 & Family 1: III-1 & $60, \mathrm{~F}$ & GD & - & ETV, LPS & $+(\mathrm{DNAH14})$ & GR \\
\hline 2 & Family 1: III-2 & $57, \mathrm{~F}$ & $C D$ & - & LPS & $+(\mathrm{DNAH14})$ & GR \\
\hline 3 & Family 1: IV-2 & $31, \mathrm{M}$ & $C D$ & - & VPS & $+($ DNAH14) & GR \\
\hline 4 & Family 2: II-2 & $38, M$ & None & $\mathrm{NI}$ & None & + (unidentified) & NC \\
\hline 5 & Family 2: III-2 & $5, \mathrm{M}$ & Macrocephaly & - & None & $+($ unidentified $)$ & NC \\
\hline 6 & Family 4: II-2 & $69, \mathrm{M}$ & GD, CD, UD & - & LPS & NP & GR \\
\hline 7 & Family 5: II-2 & $77, \mathrm{M}$ & $C D$ & - & LPS & NP & GR \\
\hline 8 & Sporadic & $63, F$ & GD, UD & $\mathrm{NI}$ & VPS & NP & GR \\
\hline 9 & Sporadic & $74, \mathrm{M}$ & GD, UD & - & LPS & NP & GR \\
\hline 10 & Sporadic & $73, \mathrm{M}$ & $\mathrm{GD}, \mathrm{CD}$ & - & LPS & NP & GR \\
\hline 11 & Sporadic & $33, \mathrm{M}$ & None & - & None & NP & NC \\
\hline \multicolumn{8}{|c|}{ w/ downward bulging 3rd ventricular floor $(n=17)$} \\
\hline 12 & Family 3: II-2 & $69, \mathrm{M}$ & $\mathrm{GD}, \mathrm{CD}, \mathrm{UD}$ & $\mathrm{NI}$ & ETV & + (unidentified) & GR \\
\hline 13 & Family $3:$ II-3 & $65, \mathrm{M}$ & $\mathrm{GD}, \mathrm{CD}, \mathrm{UD}$ & $\mathrm{NI}$ & ETV & + (unidentified) & GR \\
\hline 14 & Family 3: II-4 & $63, \mathrm{M}$ & UD & $\mathrm{NI}$ & ETV & + (unidentified) & GR \\
\hline 15 & Sporadic & $73, \mathrm{~F}$ & GD, UD & + & ETV, LPS & NP & GR \\
\hline 16 & Sporadic & $49, F$ & GD & $\mathrm{NI}$ & ETV & NP & GR \\
\hline 17 & Sporadic & $48, F$ & GD & + & ETV & NP & GR \\
\hline 18 & Sporadic & $73, \mathrm{~F}$ & GD, UD & + & ETV & NP & GR \\
\hline 19 & Sporadic & $20, M$ & None & + & None & NP & $\mathrm{NC}$ \\
\hline 20 & Sporadic & $8, \mathrm{~F}$ & Macrocephaly & + & ETV & NP & GR \\
\hline 21 & Sporadic & $68, M$ & GD, UD & + & ETV & NP & GR \\
\hline 22 & Sporadic & $6, \mathrm{M}$ & Macrocephaly & + & ETV & NP & GR \\
\hline 23 & Sporadic & $3, \mathrm{~F}$ & Macrocephaly & + & ETV & NP & GR \\
\hline 24 & Sporadic & $67, \mathrm{M}$ & GD & + & ETV, LPS & NP & GR \\
\hline 25 & Sporadic & $39, F$ & Headache & + & ETV & NP & GR \\
\hline 26 & Sporadic & $36, F$ & Headache & + & ETV & NP & GR \\
\hline 27 & Sporadic & $45, \mathrm{~F}$ & Headache & + & None & NP & NC \\
\hline 28 & Sporadic & $9, \mathrm{M}$ & Macrocephaly & + & None & $\mathrm{NP}$ & NC \\
\hline
\end{tabular}

$\mathrm{CD}=$ cognitive dysfunction; $\mathrm{GD}=$ gait disturbance; $\mathrm{GR}=$ good recovery; $\mathrm{NC}=$ no change; $\mathrm{NI}=$ no information; $\mathrm{NP}=$ not performed; prepontine membrane = membranous structures in prepontine cisterns; UD = urinary dysfunction; + = present; - = absent. 

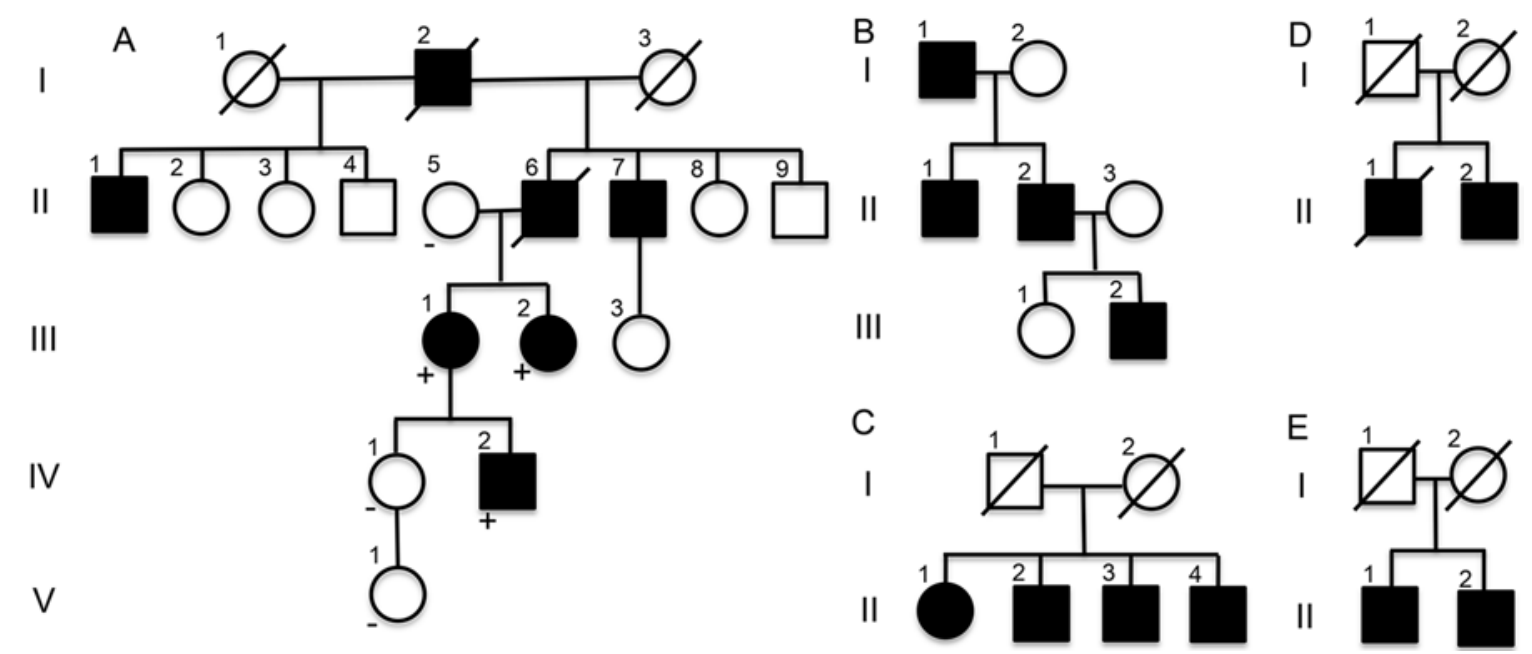

FIG. 1. Pedigrees of 5 families with PaVM defined by a wide foramen of Magendie and a large cisterna magna. A: Family 1. B: Family 2. C: Family 3. D: Family 4, E: Family 5. Filled symbols represent patients with hydrocephalus; open symbols represent unaffected individuals. Slashes indicate death. Roman numerals indicate generations. Eight patients (Family 1: III-1, III-2, and IV-2; Family 2: II-2 and III-2; Family 3: II-2, II-3, and II-4) and 4 unaffected members from 2 families (Family 1: II-5, IV-1, and V-1; Family 2: II-3) participated in copy number analysis. A: Co-segregation of the DNAH14 mutation in Family 1. + = presence of the DNAH14 deletion; - = absence of the DNAH14 deletion.

investigated, focusing on the age at disease onset, sex, earliest symptoms, the 3 major symptoms of NPH (dementia, incontinence, and gait disturbance), and surgical procedures (if performed) including ventriculoperitoneal (VP) shunting, lumboperitoneal (LP) shunting, and endoscopic third ventriculostomy (ETV).

\section{Magnetic Resonance Imaging Analysis}

Imaging was performed using a field strength of $1.5 \mathrm{~T}$ (Toshiba) or $3 \mathrm{~T}$ (Philips Healthcare). T2-weighted imaging findings (including 3D fast asymmetrical spin echo sequence [3D-FASE] and FLAIR images) were recorded. 3D-FASE is commonly used for heavily T2-weighted MR cisternography. ${ }^{28}$ In some patients, time-spatial labeling inversion pulse (time-SLIP) examinations were also performed, and CSF movement visualized as previously described. ${ }^{42}$ The shape of the third ventricle floor was examined in sagittal sections from T2-weighted images. The arachnoid membrane of the interpeduncular and prepontine cisterns was examined in 3D-FASE sagittal sections from some patients. Membranous structures in cisterns were clearly visualized by heavily T2-weighted MR cisternography. ${ }^{13}$

\section{DNA Extraction}

Genomic DNA was extracted from peripheral blood or saliva. PAXgene DNA tubes (PreAnalytiX GmbH) were used for peripheral blood sampling, and genomic DNA was extracted using a PAXgene Blood DNA kit (Qiagen) according to the manufacturer's instructions. Salivary DNA was collected using an Oragene DNA Sample Collection Kit (Oragene-DNA, Genotek).

\section{Microarray Detection of Copy Number Variation}

Genome-wide DNA copy number analysis was performed using a Cytoscan HD Array (Affymetrix) accord- ing to the manufacturer's protocol. Two patients from Family 1 (Fig. 1A), 2 patients from Family 2 (Fig. 1B), and 3 patients from Family 3 (Fig. 1C) were examined. Data were analyzed using Chromosome Analysis Suite software (ChAS; Affymetrix). The copy number variation (CNV) detection conditions were as follows: for duplications, a confidence value of $\geq 90 \%, 20$ or more contiguous probes, and $>100-\mathrm{kb}$ nucleotide length; and for deletions, a confidence value of $\geq 88 \%, 20$ or more contiguous markers, and $>10-\mathrm{kb}$ nucleotide length. ${ }^{12}$ Segmental duplications and regions registered in the Database of Genomic Variants (DGV; http://dgv.tcag.ca/dgv/app/home) were excluded as pathological CNV candidates.

\section{Quantitative Polymerase Chain Reaction (TaqMan Copy Number Assays)}

To confirm copy number quantification, TaqMan quantitative polymerase chain reaction (qPCR) using an ABI7900 real-time PCR system (Applied Biosystems) was performed on 3 patients (III-1, III-2, and IV-2) and an unaffected member (II-5) from Family 1 (Fig. 1A). Four DNAH14 probes were used: Hs 04193802_cn (exon 26), Hs 03354837_cn (exon 29), Hs 03356634_cn (exon 30), and Hs 03375696_cn (exon 39). TaqMan Copy Number Reference assay RNase P (Life Technologies) was used according to the manufacturer's instructions. CNVs were analyzed using ABI7900 Software (Applied Biosystems) and Copy Caller Software version 2.0 (Applied Biosystems).

\section{Deletion Breakpoint PCR}

Primers were designed to span the deleted region of DNAH14 based on the microarray results: forward primer 5'-TCAGGTCATTTTTCTACCACCA-3' and reverse primer 5'-CACAGATTTTTAACACACATTTGGA-3'. The PCR mixture containing ExTaq polymerase (Takara Bio) with $0.5 \mu \mathrm{M}$ of each primer, 10× ExTaq Buffer $(20 \mathrm{mM}$ 
$\mathrm{Mg}^{2+}$ plus), $2.5 \mathrm{mM}$ each dNTP, and $1 \mu$ ligated DNA was cycled 35 times at $94^{\circ} \mathrm{C}$ for 30 seconds, $60^{\circ} \mathrm{C}$ for $1 \mathrm{~min}$ ute, and $74^{\circ} \mathrm{C}$ for 1 minute, and then sequenced using an ABI3500xL Genetic Analyzer (Life Technologies) using BigDye Terminator chemistry version 3.1 (Applied Biosystems). Sequences were analyzed using the Sequencher program (Gene Codes).

\section{Double Immunofluorescence Staining}

Paraffin sections of autopsied human brains from 2 unaffected controls were double immunostained for candidate gene products. Staining was performed using a rabbit polyclonal antibody to the dynein heavy chain protein of motile cilia, DNAH14 ${ }^{30}$ (1:50; Sigma-Aldrich), or a mouse monoclonal antibody to the cilia $\alpha$-tubulin $\operatorname{protein}^{27}(1: 500$; Sigma-Aldrich). Staining was performed overnight at $4^{\circ} \mathrm{C}$. For double labeling, sections were incubated with 2 secondary antibodies for 1 hour at room temperature: Alexa 488 donkey anti-mouse IgG (Molecular Probes, Inc.) and Alexa 594 donkey anti-rabbit IgG (Molecular Probes, Inc.). Nuclei were stained using ProLong Gold Antifade Reagent with 4',6-diamidino-2-phenylindole (Molecular Probes, Inc.). Sections were viewed, and images were captured using a Leica TCS SP5 confocal microscope (Leica Microsystems) with Leica Application Suite Advanced Fluorescence Lite 2.4.1 imaging-processing software (Leica Microsystems).

\section{Results \\ Clinical Course}

The most common symptom in adult $\mathrm{PaVM}$ patients was gait disturbance (57\%), although incontinence (39\%) and cognitive dysfunction (30\%) were also common (Table 1). Five affected children exhibited macrocranium. Hydrocephalus was incidentally diagnosed in 3 patients based on MR imaging analysis.

\section{MR Imaging Analysis}

All patients exhibited ventricular dilation (Evans' index $0.38 \pm 0.06)$. Tightness of high-convexity sulci was not detected in PaVM patients (Fig. 2C and $\mathrm{H}$ ). In patients with time-SLIP sequences, CSF flow through the aqueduct and foramen of Magendie was observed (Video 1, Patient 1; Video 2, Patient 17).

VIDEO 1. Patient 1. Time-SLIP examinations from a patient without bulging third ventricular floors. CSF flow was observed through the aqueduct and foramen of Magendie. CSF movement was also showed in the prepontine cistern. Copyright Hiroshi Kageyama. Published with permission. Click here to view.

VIDEO 2. Patient 17. Time-SLIP examinations from a patient with bulging third ventricular floors. CSF movement was restricted in the prepontine cistern. Copyright Hiroshi Kageyama. Published with permission. Click here to view.

Patients were divided into 2 groups by the presence or absence of a downwardly bulging third ventricular floor (Table 1). Seventeen patients showed a downward bulging third ventricular floor. Of these, all patients who were checked had membranous structures in the prepontine cistern on 3D-FASE paramedian sagittal sections (Fig. $2 \mathrm{~K})$. Patients without a downward bulging third ventricu- lar floor lacked these membranes. We did not obtain 3DFASE paramedian sagittal sections from 5 patients; therefore, we were unable to check for membranes in these patients. Interestingly, CSF movement was restricted in the prepontine cistern of patients with bulging third ventricular floors (Video 2). Patients without bulging third ventricular floors showed CSF movement in the prepontine cistern (Video 1).

\section{Therapeutic Procedures}

Patients with a downward bulging third ventricular floor were treated with ETV (14 of 17 patients). Of these patients, LP shunt treatment was also performed in 2 patients after ETV because the ETV was insufficient. Patients without a downward bulging third ventricular floor were treated with an LP shunt or VP shunt (8 of 11 patients). Six patients were under observation but did not undergo surgery.

\section{CNV Detection}

Microarray analysis identified CNV that did not overlap with segmental duplications. In Family 1, a 322-kb heterozygous deletion at 1q42.12 (UCSC Genome Browser, chromosome 1: 225,186,483-225,509,460) was detected in 2 patients (III-1 and III-2), but was absent in the mother of these patients (II-5) and 2 healthy controls. No pathogenic CNVs were detected in Family 2 or Family 3 (Fig. 3A).

All 3 patients in Family 1 carried this deletion, as shown by qPCR analysis (Fig. 3B). PCR products specific for the deleted allele were amplified in all 3 patients, but not in 3 unaffected members of Family 1 (Fig. 3C). Sequencing the PCR products revealed that the deletion was $319,330 \mathrm{bp}$ in size (UCSC Genome Browser, chromosome 1: $225,190,746-225,510,076$ bp) (Fig. 3D). This deletion has not been previously reported in DGV, but at least 5 CNVs partially overlapped with this deletion that incorporates DNAH14 exons (Fig. 4).

\section{Immunostaining of Autopsied Brain}

Staining of the autopsied control brains revealed that DNAH14 specifically localized to ependymal cells and choroid plexus epithelial cells (Alexa594; Fig. 5, red), and $\alpha$-tubulin localized to ependymal cells, choroid plexus epithelial cells, and ependymal cilia at the interface between the parenchyma and CSF space.

\section{Discussion}

Our PaVM patients presented with unique clinical features. Although PaVM shared major symptoms with iNPH, age of onset, surgical options, and familial accumulation were different (Table 2). Age at onset in our patient group $(56.0 \pm 16.7$ years) was younger than that reported for iNPH (75 years)..$^{24}$ The majority of iNPH patients typically show ventriculomegaly with tightness of high-convexity sulci. ${ }^{2}, 16,18$ This type of iNPH is currently called disproportionately enlarged subarachnoid space hydrocephalus (DESH). ${ }^{2,16}$ Although components of adultonset $\mathrm{PaVM}$ may be included in iNPH, PaVM patients show distinct characteristics compared with those with DESH (Table 2). 

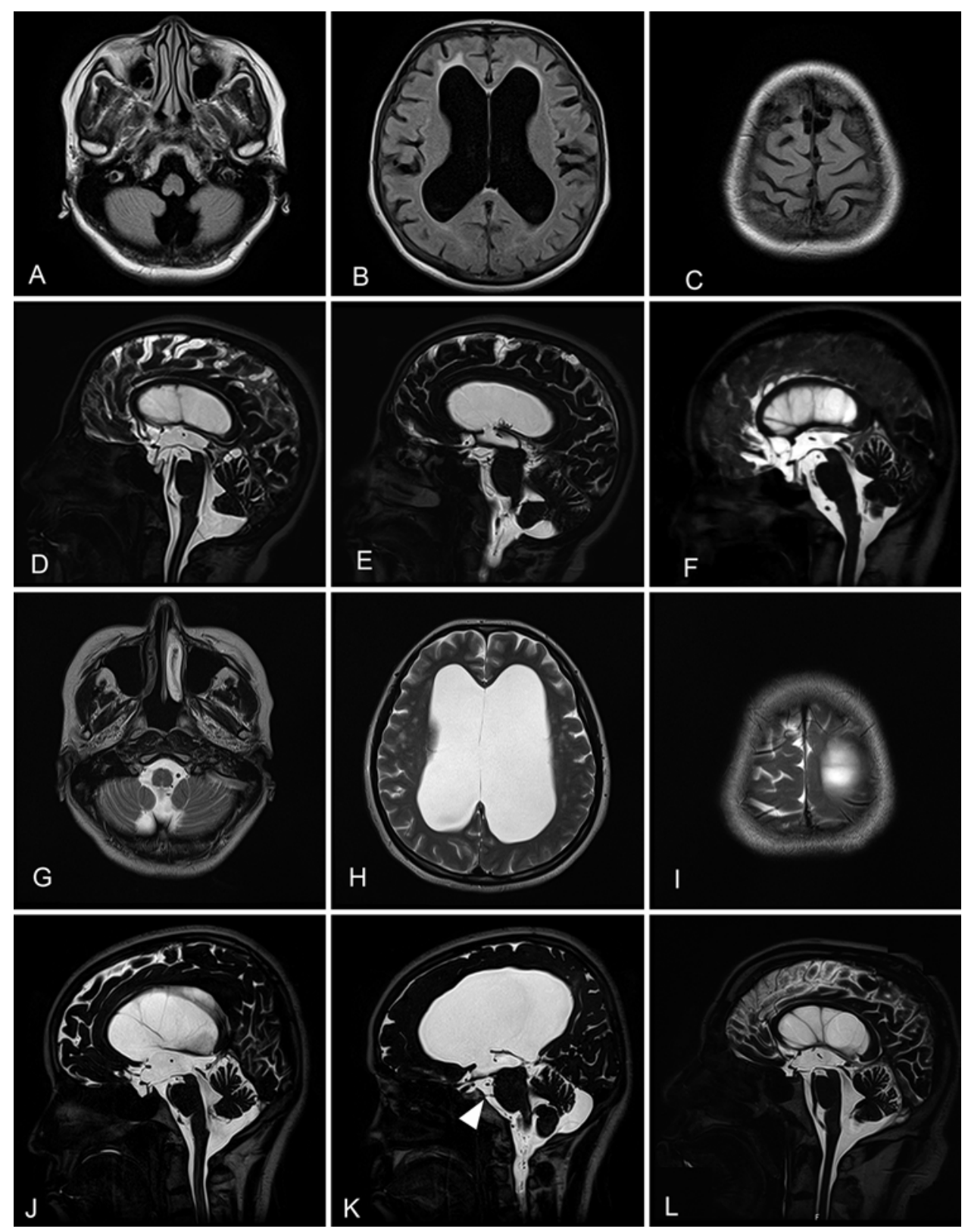

FIG. 2. MR images from PaVM patients. Patients $1(\mathbf{A}-\mathbf{E})$ and $2(\mathrm{~F})$ do not have a downward bulging third ventricular floor. Patients 17 (G-K) and 21 (L) have a downward bulging third ventricular floor (Table 1). A-C and G-I: Axial FLAIR (A-C) and T2-weighted $(G-l)$ sections showing a wide foramen of Magendie and dilation of the lateral ventricles. High convexity and the medial subarachnoid space were not tight, unlike in the majority of iNPH cases. ${ }^{2,16,18} \mathrm{D}, \mathrm{F}, \mathrm{J}$, and L: Midsagittal 3D-FASE sections showing a wide foramen of Magendie and large cisterna magna. K: A paramedian sagittal 3D-FASE section showing a thick membranous structure in the prepontine cistern (arrowhead). E: A paramedian section without this membrane.

Twelve patients underwent ETV treatment alone. Kehler et al. showed that downward bulging third ventricular floors are associated with effectiveness of ETV treatment in cases of communicating hydrocephalus. ${ }^{19}$ Bulging reflects the pressure gradient between the third ventricle and interpeduncular/prepontine cisterns. ${ }^{20}$ In our patients, many with bulging third ventricular floors had prepontine membranes. Because these membranes might be associated with the pressure gradient, we removed them through the third ventricle stoma. In the present study, LP shunt or VP shunt treatment alone was performed in 7 patients without downward bulging third ventricular floors. LP shunt treatment was also performed after ETV because ETV treatment was not effective. Thus, we may have included 2 separate clinical entities with the same radiological features, except for the shape of the third ventricle.

Our MR imaging analysis suggests that PaVM in the present study resembles Blake's pouch cyst. ${ }^{8}$ This incorporates the spectrum of posterior fossa cysts and cyst-like malformations that were first reported in $1996^{36}$ and includes the following radiological characteristics: tetraventricular hydrocephalus, infra- or retrocerebellar cyst lo- 

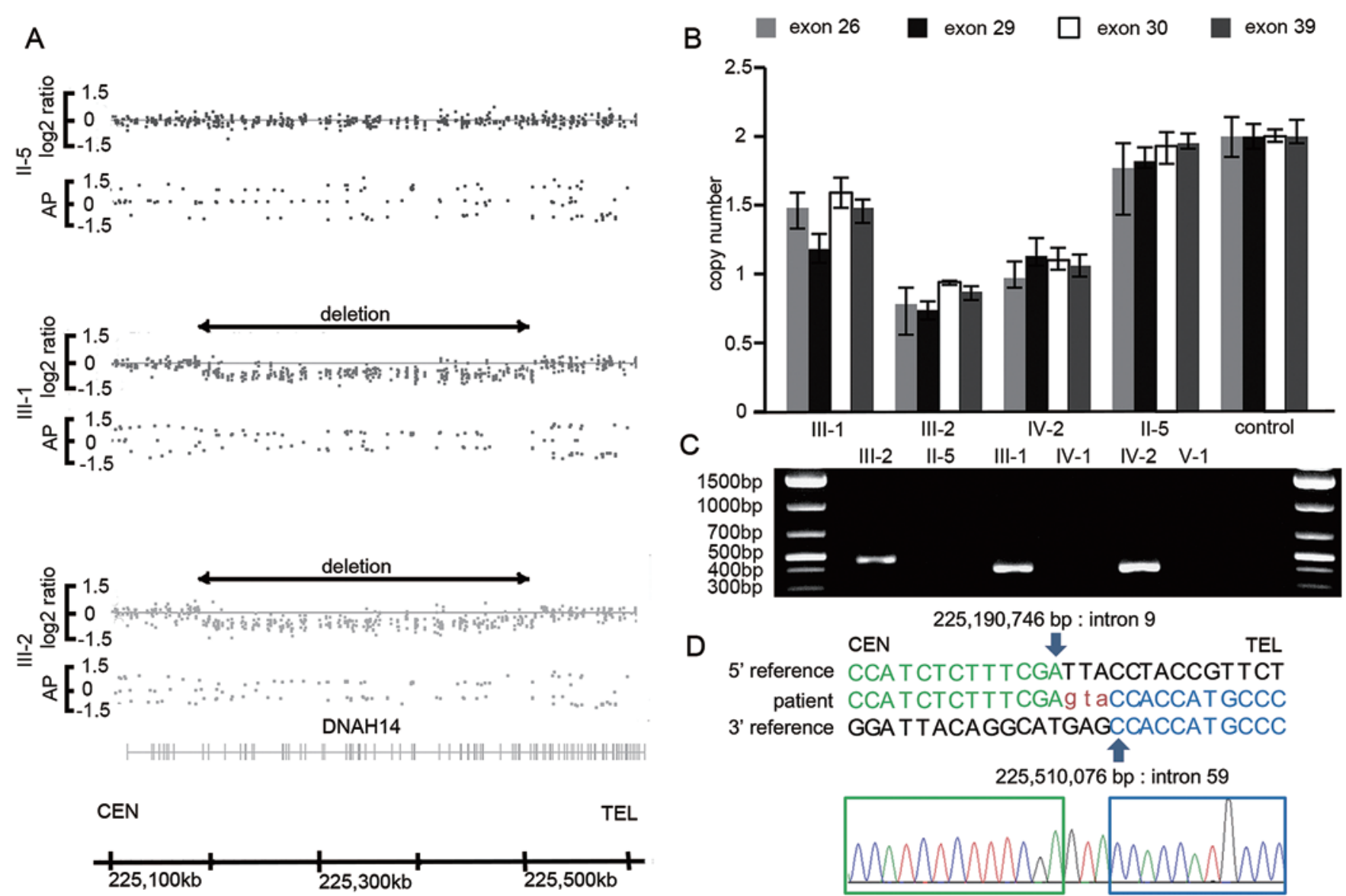

FIG. 3. DNAH14 deletion in Family 1. A: Microarray analysis of II-5 (upper), Patient 1 (III-1) (center), and Patient 2 (III-2) (lower). Heterozygous DNAH14 deletion was identified in Patients 1 and 2. The bidirectional horizontal arrow indicates the 322-kb deletion involving DNAH14. In each microarray panel, the upper and lower tracks show $\log _{2}$ ratios $(2$ copy $=0)$ and allele peaks $(A P)$, respectively. Three allele peaks ( $A A, A B$, and $B B$ ) were observed in the normal copy region, whereas only 2 peaks ( $A A$ and $B B$ ) (no heterozygous alleles) were observed in the deleted region. B: Quantitative PCR. DNAH14 copy numbers (CNs) were confirmed with 4 probe/primer sets. All patients in Family $1(\mathrm{III}-1$, III-2, and IV-2) showed $\mathrm{CN}$ loss $(\mathrm{CN}=1)$ in this area. An unaffected member of Family 1 (II-5) and a normal control showed no deletion. C: Deletion breakpoint PCR. PCR products (477 bp) were only obtained for patients from Family 1. Co-segregation of affected status and DNAH14 deletion was confirmed in Family 1 (1\% agarose gel). D: Sanger sequencing of the deletion breakpoint. Upper, middle, and lower sequence strands show proximal, deleted, and distal chromosomal sequences, respectively. Identical sequences of proximal regions are highlighted in green (also shown as a green box in the electropherogram). Those in distal sequences are colored blue (also shown as a blue box in the electropherogram). Three inserted nucleotides of unknown origin are highlighted in magenta. Sequencing confirmed a 319,330 bp deletion (UCSC genome browser, Feb 2009; chromosome 1: 225,190,746-225,510,076 bp) in only the affected members of Family 1 (III-1, III-2, and IV-2). Arrows indicate confirmed proximal and distal deletion breakpoints.

calization, nonrotated cerebellar vermis, cystic dilation of the fourth ventricle without cisternal communication, and degree of compression on medial cerebellar hemispheres. ${ }^{8}$

Two features are different, however. First, Blake's pouch cyst involves cystic dilation of the fourth ventricle, which was not seen in our PaVM patients. Second, Blake's pouch cyst is an obstructive hydrocephalus without cisternal communication, but our time-SLIP examinations and effectiveness of LP shunting suggest that PaVM is a communicating hydrocephalus.

Familial occurrence of iNPH is uncommon, having previously been reported only 4 times. ${ }^{25,32,34,43}$ No causative mutations were identified in these families. In the present study, the observed familial occurrence suggests possible involvement of a genetic factor. Copy number analysis identified a deletion involving $D N A H 14$, which encodes an axonemal dynein heavy chain protein involved in microtubule binding and conversion of adenosine triphosphate energy to force, chiefly in motile cilia.

According to the BioGPS database (http://biogps.org), DNAH14 is expressed throughout the human body. Its discrete localization in the ventricular system and subarachnoid space has never been shown. Here, our immunohistochemical analysis revealed DNAH14 to be abundantly localized in ependymal cells and choroid plexus epithelial cells. This suggests that DNAH14 deletion may affect physiological function of cilia during hydrocephalus pathogenesis. CSF production or absorption may be disturbed in the ependyma or choroid plexus. However, the exact pathophysiological details of the hydrocephalus associated with motile cilia in our patients remain unclear.

Dysfunction in dynein heavy chain families is asso- 


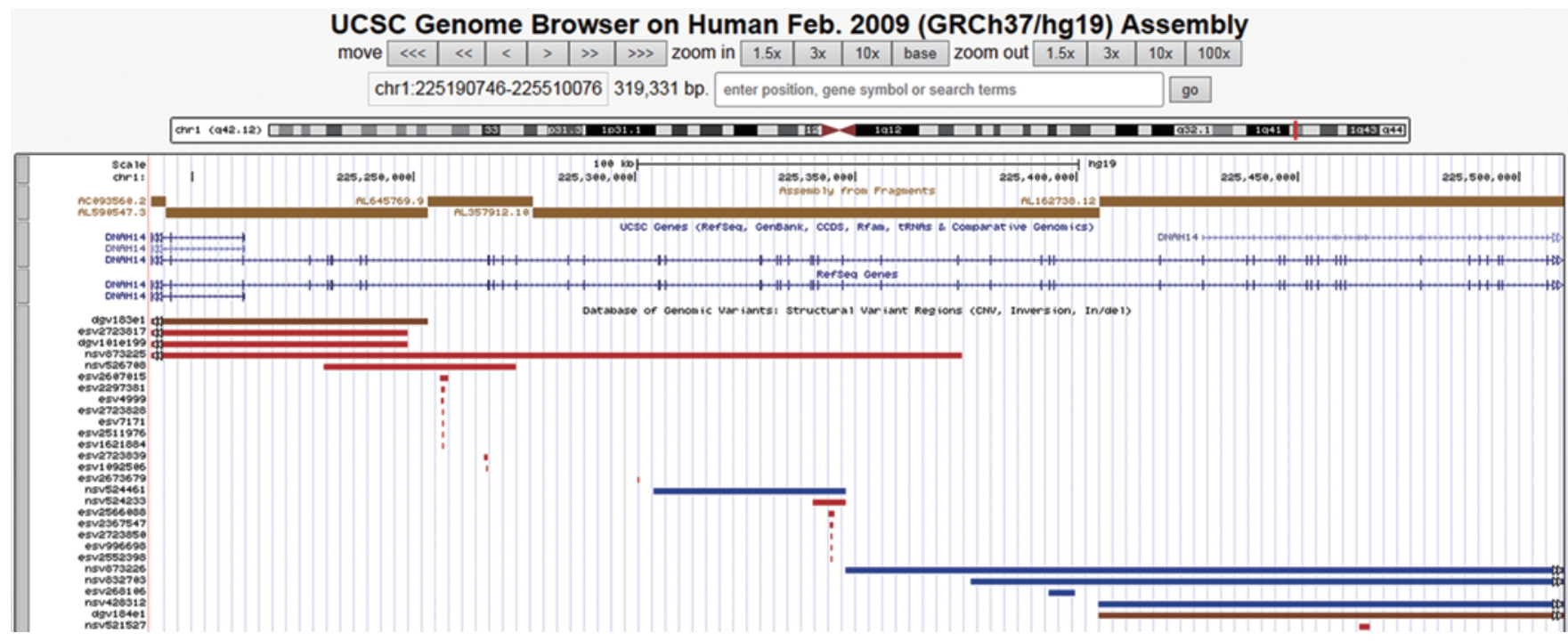

FIG. 4. Copy number variants within the DNAH14 deletion area of Family 1. Registered CNVs for apparently normal phenotypes in DGV are shown in the UCSC Genome Browser (http://genome.ucsc.edu/cgi-bin/hgGateway) (chromosome arm 1q42.12: $225,190,746-225,510,076$ bp). Red and blue bars indicate deletions and duplications found in controls, respectively.

ciated with primary ciliary dyskinesia, ${ }^{26}$ which presents with respiratory infection, situs inversus, and sometimes hydrocephalus. Cases of primary ciliary dyskinesia-associated hydrocephalus in humans have previously been reported, although the causative mutations have not been identified. . $, 6,10,14,21,31,37,39^{2}$

We observed a pathogenic CNV involving DNAH14 in only one family in the present study, although it is possible that techniques such as next-generation sequencing would identify other hydrocephalus-associated mutant genes in these patients, including those in Family 1. CNV involving DNAHI4 had been reported in DGV (Fig. 4). Many animal models of hydrocephalus show mutations in genes associated with motile cilia, $,, 5,7,9,11,15,17,23,33,35,38,41,44$ including other dynein heavy chain genes. It is conceivable that hydrocephalus is not recognized unless imaging studies are performed. Additional studies are required to determine the relationship between hydrocephalus and DNAH14 abnormalities.

\section{Conclusions}

Panventriculomegaly with a wide foramen of Magendie and a large cisterna magna exhibits unique characteris-

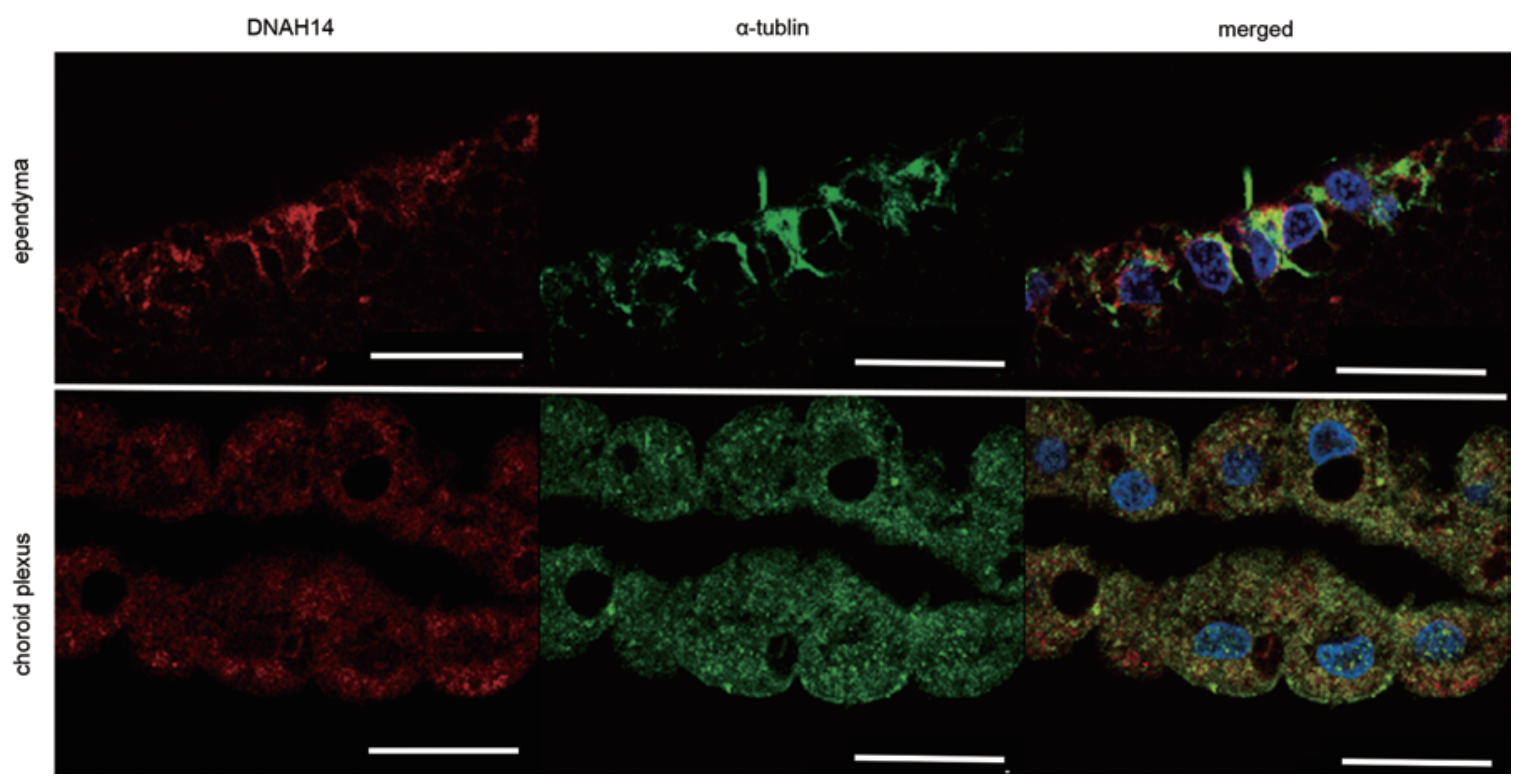

FIG. 5. Double-immunofluorescence staining with DNAH14 and $\alpha$-tubulin antibodies in autopsied brain specimens from unaffected individuals. DNAH14-positive (red), $\alpha$-tubulin positive (green), and double-positive cells (merged) are indicated. Upper and lower panels show the ependyma and choroid plexus, respectively. Bars $=20 \mu \mathrm{m}$. 
TABLE 2. Imaging and genetic findings comparing PaVM, iNPH, and aqueductal stenosis

\begin{tabular}{|c|c|c|c|c|}
\hline Finding & $\mathrm{Pa}$ & & DESH-iNPH & Aqueductal Stenosis \\
\hline Bulging of the 3rd ventricular floor & - & + & - & + \\
\hline Ventricular dilation & Tetra & Tetra & Tetra & Lateral \& 3rd ventricles \\
\hline Aqueduct & Open & Open & Open & Occlusion \\
\hline Tightness of high convexity sulci & - & - & + & - \\
\hline Familial accumulation & 4 families (7/11 patients) & 1 family (3/17 patients) & Rare & $\begin{array}{l}\text { X-linked (MIM307000) }\left(1 / 30,000 \text { males) }{ }^{1,40}\right. \\
\text { AR (MIM236635) } 8 \text { families } 22,40\end{array}$ \\
\hline Gene mutations in human & DNAH14 & Unidentified & Unidentified & L1CAM (X-linked) $)^{1,40}$ \\
\hline
\end{tabular}

$\mathrm{AR}=$ autosomal recessive; tetra = tetraventricular dilation.

tics distinct from other types of hydrocephalus with NPH symptoms (e.g., DESH, aqueductal stenosis, and Blake's pouch cyst); specifically, imaging findings, younger age at onset, tractability to ETV treatment, and familial accumulation. Thus, PaVM likely belongs to a separate clinical entity. PaVM can be divided into 2 subcategories: with or without bulging of the third ventricular floor. A portion of PaVM cases may be associated with genetic factors that include a DNAH14 abnormality.

\section{Acknowledgments}

This work was supported by the Ministry of Health, Labour and Welfare of Japan; the Japan Society for the Promotion of Science (a Grant-in-Aid for Scientific Research [B], and a Grant-in-Aid for Scientific Research [A]); the Takeda Science Foundation; the Fund for Creation of Innovation Centers for Advanced Interdisciplinary Research Areas Program in the Project for Developing Innovation Systems; the Strategic Research Program for Brain Sciences; and a Grant-in-Aid for Scientific Research on Innovative Areas (Transcription Cycle) from the Ministry of Education, Culture, Sports, Science and Technology of Japan.

The microarray analysis was supported in part by the Laboratory of Molecular and Biochemical Research (Research Support Center, Juntendo University Graduate School of Medicine, Tokyo, Japan) and the Juntendo University Research Institute for Diseases of Old Age (Tokyo, Japan). The confocal microscope study was supported in part by the Division of Biomedical Imaging Research (Research Support Center, Juntendo University Graduate School of Medicine, Tokyo, Japan). In particular, we thank M. Kunichika from this division for preparing the autopsied brain paraffin sections.

\section{References}

1. Adle-Biassette H, Saugier-Veber P, Fallet-Bianco C, Delezoide AL, Razavi F, Drouot N, et al: Neuropathological review of 138 cases genetically tested for X-linked hydrocephalus: evidence for closely related clinical entities of unknown molecular bases. Acta Neuropathol 126:427-442, 2013

2. Akiguchi I, Shirakashi Y, Budka H, Watanabe Y, Watanabe T, Shiino A, et al: Disproportionate subarachnoid space hydrocephalus-outcome and perivascular space. Ann Clin Transl Neurol 1:562-569, 2014

3. al-Shroof M, Karnik AM, Karnik AA, Longshore J, Sliman NA, Khan FA: Ciliary dyskinesia associated with hydrocephalus and mental retardation in a Jordanian family. Mayo Clin Proc 76:1219-1224, 2001

4. Appelbe OK, Bollman B, Attarwala A, Triebes LA, MunizTalavera H, Curry DJ, et al: Disruption of the mouse Jhy gene causes abnormal ciliary microtubule patterning and juvenile hydrocephalus. Dev Biol 382:172-185, 2013
5. Banizs B, Pike MM, Millican CL, Ferguson WB, Komlosi P, Sheetz J, et al: Dysfunctional cilia lead to altered ependyma and choroid plexus function, and result in the formation of hydrocephalus. Development 132:5329-5339, 2005

6. Berlucchi M, de Santi MM, Bertoni E, Spinelli E, Timpano S, Padoan R: Ciliary aplasia associated with hydrocephalus: an extremely rare occurrence. Eur Arch Otorhinolaryngol 269:2295-2299, 2012

7. Chandrasekar G, Vesterlund L, Hultenby K, Tapia-Páez I, Kere J: The zebrafish orthologue of the dyslexia candidate gene DYX1C1 is essential for cilia growth and function. PLoS One 8:e63123, 2013

8. Cornips EM, Overvliet GM, Weber JW, Postma AA, Hoeberigs CM, Baldewijns MM, et al: The clinical spectrum of Blake's pouch cyst: report of six illustrative cases. Childs Nerv Syst 26:1057-1064, 2010

9. Davy BE, Robinson ML: Congenital hydrocephalus in hy3 mice is caused by a frameshift mutation in Hydin, a large novel gene. Hum Mol Genet 12:1163-1170, 2003

10. De Santi MM, Magni A, Valletta EA, Gardi C, Lungarella G: Hydrocephalus, bronchiectasis, and ciliary aplasia. Arch Dis Child 65:543-544, 1990

11. Fernandez-Gonzalez A, Kourembanas S, Wyatt TA, Mitsialis SA: Mutation of murine adenylate kinase 7 underlies a primary ciliary dyskinesia phenotype. Am J Respir Cell Mol Biol 40:305-313, 2009

12. Fukai R, Ochi N, Murakami A, Nakashima M, Tsurusaki Y, Saitsu H, et al: Co-occurrence of 22q11 deletion syndrome and HDR syndrome. Am J Med Genet A 161A:2576-2581, 2013

13. Fushimi Y, Miki Y, Ueba T, Kanagaki M, Takahashi T, Yamamoto A, et al: Liliequist membrane: three-dimensional constructive interference in steady state MR imaging. Radiology 229:360-365, 2003

14. Greenstone MA, Jones RW, Dewar A, Neville BG, Cole PJ: Hydrocephalus and primary ciliary dyskinesia. Arch Dis Child 59:481-482, 1984

15. Hagenlocher C, Walentek P, M Ller C, Thumberger T, Feistel $\mathrm{K}$ : Ciliogenesis and cerebrospinal fluid flow in the developing Xenopus brain are regulated by foxj1. Cilia 2:12, 2013

16. Hashimoto M, Ishikawa M, Mori E, Kuwana N: Diagnosis of idiopathic normal pressure hydrocephalus is supported by MRI-based scheme: a prospective cohort study. Cerebrospinal Fluid Res 7:18, 2010

17. Ibañez-Tallon I, Pagenstecher A, Fliegauf M, Olbrich H, Kispert A, Ketelsen UP, et al: Dysfunction of axonemal dynein heavy chain Mdnah5 inhibits ependymal flow and reveals a novel mechanism for hydrocephalus formation. Hum Mol Genet 13:2133-2141, 2004

18. Jaraj D, Rabiei K, Marlow T, Jensen C, Skoog I, Wikkelsø C: Prevalence of idiopathic normal-pressure hydrocephalus. Neurology 82:1449-1454, 2014

19. Kehler U, Gliemroth J: Extraventricular intracisternal obstructive hydrocephalus - a hypothesis to explain successful 
3rd ventriculostomy in communicating hydrocephalus. Pediatr Neurosurg 38:98-101, 2003

20. Kehler U, Regelsberger J, Gliemroth J, Westphal M: Outcome prediction of third ventriculostomy: a proposed hydrocephalus grading system. Minim Invasive Neurosurg 49:238-243, 2006

21. Kosaki K, Ikeda K, Miyakoshi K, Ueno M, Kosaki R, Takahashi D, et al: Absent inner dynein arms in a fetus with familial hydrocephalus-situs abnormality. Am J Med Genet A 129A:308-311, 2004

22. Lapunzina P, Delicado A, de Torres ML, Mor MA, Pérez-Pacheco RF, Lópes PI: Autosomal recessive hydrocephalus due to aqueduct stenosis: report of a further family and implications for genetic counselling. J Matern Fetal Neonatal Med 12:64-66, 2002

23. Lee L, Campagna DR, Pinkus JL, Mulhern H, Wyatt TA, Sisson $\mathrm{JH}$, et al: Primary ciliary dyskinesia in mice lacking the novel ciliary protein Pcdp1. Mol Cell Biol 28:949-957, 2008

24. Marmarou A, Young HF, Aygok GA, Sawauchi S, Tsuji O, Yamamoto T, et al: Diagnosis and management of idiopathic normal-pressure hydrocephalus: a prospective study in 151 patients. J Neurosurg 102:987-997, 2005

25. McGirr A, Cusimano MD: Familial aggregation of idiopathic normal pressure hydrocephalus: novel familial case and a family study of the NPH triad in an iNPH patient cohort. J Neurol Sci 321:82-88, 2012

26. Mitchison HM, Schmidts M, Loges NT, Freshour J, Dritsoula A, Hirst RA, et al: Mutations in axonemal dynein assembly factor DNAAF3 cause primary ciliary dyskinesia. Nat Genet 44:381-389, S1-S2, 2012

27. Mohri H, Inaba K, Ishijima S, Baba SA: Tubulin-dynein system in flagellar and ciliary movement. Proc Jpn Acad, Ser B, Phys Biol Sci 88:397-415, 2012

28. Naganawa S, Koshikawa T, Fukatsu H, Ishigaki T, Fukuta T: MR cisternography of the cerebellopontine angle: comparison of three-dimensional fast asymmetrical spin-echo and three-dimensional constructive interference in the steadystate sequences. AJNR Am J Neuroradiol 22:1179-1185, 2001

29. Oi S, Shimoda M, Shibata M, Honda Y, Togo K, Shinoda M, et al: Pathophysiology of long-standing overt ventriculomegaly in adults. J Neurosurg 92:933-940, 2000

30. Pazour GJ, Agrin N, Walker BL, Witman GB: Identification of predicted human outer dynein arm genes: candidates for primary ciliary dyskinesia genes. J Med Genet 43:62-73, 2006

31. Picco P, Leveratto L, Cama A, Vigliarolo MA, Levato GL, Gattorno M, et al: Immotile cilia syndrome associated with hydrocephalus and precocious puberty: a case report. Eur J Pediatr Surg 3 (Suppl 1):20-21, 1993

32. Portenoy RK, Berger A, Gross E: Familial occurrence of idiopathic normal-pressure hydrocephalus. Arch Neurol 41:335-337, 1984

33. Sironen A, Kotaja N, Mulhern H, Wyatt TA, Sisson JH, Pavlik JA, et al: Loss of SPEF2 function in mice results in spermatogenesis defects and primary ciliary dyskinesia. Biol Reprod 85:690-701, 2011

34. Takahashi Y, Kawanami T, Nagasawa H, Iseki C, Hanyu H, Kato T: Familial normal pressure hydrocephalus (NPH) with an autosomal-dominant inheritance: a novel subgroup of NPH. J Neurol Sci 308:149-151, 2011

35. Tarkar A, Loges NT, Slagle CE, Francis R, Dougherty GW, Tamayo JV, et al: DYX1C1 is required for axonemal dynein assembly and ciliary motility. Nat Genet 45:995-1003, 2013

36. Tortori-Donati P, Fondelli MP, Rossi A, Carini S: Cystic malformations of the posterior cranial fossa originating from a defect of the posterior membranous area. Mega cisterna magna and persisting Blake's pouch: two separate entities. Childs Nerv Syst 12:303-308, 1996
37. Vieira JP, Lopes P, Silva R: Primary ciliary dyskinesia and hydrocephalus with aqueductal stenosis. J Child Neurol 27:938-941, 2012

38. Vogel P, Read R, Hansen GM, Freay LC, Zambrowicz BP, Sands AT: Situs inversus in Dpcd/Poll- ${ }^{-/}, \mathrm{Nme}^{-/-}$, and Pkd111 ${ }^{-/}$ mice. Vet Pathol 47:120-131, 2010

39. Wessels MW, den Hollander NS, Willems PJ: Mild fetal cerebral ventriculomegaly as a prenatal sonographic marker for Kartagener syndrome. Prenat Diagn 23:239-242, 2003

40. Williams CA, Dagli A, Battaglia A: Genetic disorders associated with macrocephaly. Am J Med Genet A 146A:20232037, 2008

41. Wilson GR, Wang HX, Egan GF, Robinson PJ, Delatycki MB, O'Bryan MK, et al: Deletion of the Parkin co-regulated gene causes defects in ependymal ciliary motility and hydrocephalus in the quaking viable mutant mouse. Hum Mol Genet 19:1593-1602, 2010

42. Yamada S, Miyazaki M, Kanazawa H, Higashi M, Morohoshi Y, Bluml S, et al: Visualization of cerebrospinal fluid movement with spin labeling at MR imaging: preliminary results in normal and pathophysiologic conditions. Radiology 249:644-652, 2008

43. Zhang J, Williams MA, Rigamonti D: Heritable essential tremor-idiopathic normal pressure hydrocephalus (ETINPH). Am J Med Genet A 146A:433-439, 2008

44. Zhou J, Yang F, Leu NA, Wang PJ: MNS1 is essential for spermiogenesis and motile ciliary functions in mice. PLoS Genet 8:e1002516, 2012

\section{Disclosures}

The authors report no conflict of interest concerning the materials or methods used in this study or the findings specified in this paper.

\section{Author Contributions}

Conception and design: Kageyama, Miyajima, Miyake, Nishiyama, Matsumoto, Arai. Acquisition of data: Kageyama, Miyajima, Ogino, Nakajima, Shimoji, Fukai. Analysis and interpretation of data: Kageyama, Miyajima, Ogino, Fukai, Miyake, Matsumoto. Drafting the article: Kageyama, Miyajima, Ogino, Fukai, Miyake, Nishiyama, Matsumoto. Critically revising the article: Kageyama, Miyajima, Miyake, Matsumoto. Reviewed submitted version of manuscript: Kageyama, Miyajima, Miyake, Matsumoto, Arai. Approved the final version of the manuscript on behalf of all authors: Kageyama. Statistical analysis: Kageyama. Administrative/technical/material support: Ogino, Nakajima, Shimoji, Fukai, Miyake, Nishiyama, Matsumoto. Study supervision: Miyajima, Nakajima, Shimoji, Miyake, Nishiyama, Matsumoto, Arai.

\section{Supplemental Information \\ Videos}

Video 1. https://vimeo.com/140217142.

Video 2. https://vimeo.com/140219703.

\section{Previous Presentations}

This material was presented in abstract form at Hydrocephalus 2015 (The Seventh Meeting of the International Society for Hydrocephalus and CSF Disorders), held in Banff, Alberta, Canada, September 19-21, 2015.

\section{Correspondence}

Hiroshi Kageyama, Department of Neurosurgery, Kuki General Hospital, Kamihayami 418-1, Kuki, Saitama 346-0021, Japan. email: kageyamahiroshi29@gmail.com. 